\title{
Grundlagen von Coaching und diesem Forschungsvorhaben
}

Coaching ist in aller Munde. Dass sich Sportlerinnen oder Führungskräfte coachen lassen, ist seit Jahren üblich. Immer häufiger nehmen aber auch Fachkräfte ohne Führungsaufgaben Coaching in Anspruch, ebenso Patienten, Lehrerinnen oder erwerbslose Menschen. Und Coaching verbreitet sich weiter - auf immer neue Bereiche. Schon heute gibt es Coaching für Kinder oder Jugendliche; es dürfte nur eine Frage der Zeit sein, bis entsprechende Angebote zum Beispiel auch für Flüchtlinge entwickelt werden - falls es sie nicht schon gibt.

Trotz der rasanten Verbreitung von Coaching, trotz aller Professionalisierungsbemühungen, die sich etwa in der Entwicklung von Qualitätsstandards durch Coaching-Verbände zeigen: Ein breiter Konsens zur Frage, was Coaching sei, ist nicht in Sicht. In diesem Spannungsfeld positioniert sich die vorliegende Arbeit und möchte einen Beitrag zur Professionalisierung von Coaching leisten. $\mathrm{Zu}$ diesem Zweck wird - auf der Grundlage einer systematischen Analyse und Bewertung der aktuellen Coaching-Forschung - ein Vorschlag für die Erweiterung der prozessorientierten Coaching-Forschung formuliert. Das zweite Ziel der Arbeit besteht darin, aus einer prozessorientierten Coaching-Forschungslandkarte, welche die Coaching-Wissenschaft hoffentlich inspirieren wird, Ideen zu entwickeln, wie faktische Coaching-Verläufe - mit Blick auf «bedeutsame Momente» - untersucht werden können.

In diesem Einleitungskapitel tasten wir uns zunächst ans Thema der Studie heran. Dazu werden die historischen Wurzeln von Coaching und seine ursprüngliche Etablierung als Format der Personal- und Führungsentwicklung beleuchtet.

(C) Der/die Autor(en) 2019

R. Wegener, Bedeutsame Momente im Coaching, https://doi.org/10.1007/978-3-658-25687-6_1 
Im zweiten Abschnitt werden die darauf folgende Weiterverbreitung von Coaching und deren gesellschaftlicher Kontext beschrieben; im dritten geht es um die Frage der Professionalisierung von Coaching. Im vierten Abschnitt wird Coaching als Beratungsformat begrifflich bestimmt und die der Arbeit zugrunde gelegte Definition hergeleitet. Im letzten Abschnitt geht es um Ziele, Vorgehensweise und Aufbau der vorliegenden Arbeit.

\subsection{Ursprünge von Coaching}

\subsubsection{Ideengeschichtlicher und etymologischer Ursprung}

Obschon vor dem 19. Jahrhundert noch keine Texte zu Coaching verfasst wurden, gibt Garvey (2011, S. 66) zu bedenken, dass die dem Coaching zugrunde liegende Idee nicht sonderlich neu sei. Dazu verweist er in seiner ideengeschichtlichen Rekonstruktion auf Autoren und Autorinnen, die den Ursprung von Coaching bei den Griechen («classic times») sehen, insbesondere im «Sokratischen Dialog» (Brunner 1998; de Haan 2008a) - oder gar in der Steinzeit (McDermott \& Jago 2005; Zeus \& Skiffington 2000). Solche Einschätzungen sieht Garvey in der verbreiteten Annahme begründet, dass Menschen einander seit jeher geholfen hätten, wichtige Tätigkeiten zu erlernen und darin besser zu werden - so etwa beim Werfen von Steinen oder beim Herstellen von Äxten. Nach Garvey ist es weiter der in dieser Idee des Helfens vorhandene Leistungsbezug, der gewisse Gegenwartsautoren (Starr 2002; Wilson 2007) dazu veranlasst hat, den historischen Ursprung von Coaching im Sport zu sehen (Garvey 2011, S. 66).

Sprachlich hat Coaching seine Wurzeln im Ungarischen: «Coach» geht zurück auf die im Norden Ungarns liegende Ortschaft Kocs (Cox, Bachkirova \& Clutterbuck 2010, S. 2). Dort wurden im 15. Jahrhundert spezielle Pferdefuhrwerke hergestellt (Hartmann 2004; Tonhäuser 2010). Hartmann (2004, S. 16) beschreibt, wie mit der Zeit aus den «Wagen von Kocs» schlicht «Kocsi» wurden - ein Wort, das im 16. Jahrhundert, so die Vermutung, durch ungarische Emigranten und Emigrantinnen in weitere europäische Sprachen übertragen und adaptiert wurde. Im Deutschen wurde aus «Kocsi» «Kutsche», im Französischen «coche». Weiter wird vermutet, dass das «Kutschengängigmachen» eines Pferdes mit «to coach a horse» umschrieben und dann die Begriffe «coach» und «coachman» - für Kutscher eingeführt wurden (Hartmann 2004, S. 16). So nannte man diejenigen, die die Pferde «kutschengängig» machten.

Coaching als Wort und Verb taucht dann in William Makepeace Thackerays Novelle vom Gentleman Arthur Pendennis auf, die 1849 erschien. Der Held der 
Geschichte ist auf dem Land geboren und will nach London, um dort seinen Platz in der Gesellschaft zu finden. Der Begriff erscheint in der Novelle eher beiläufig, in der Gestalt eines Wortspiels: Coaching als Beschreibung einer räumlichen Bewegung von A nach B und auch, um akademische Leistungen zu erreichen (Garvey 2011, S. 67), Letzteres ganz im Sinne des schon erwähnten Leistungsbezugs.

Im 19. Jahrhundert findet der Begriff «Coaching» dann weitere Verwendung, so etwa im Zusammenhang mit sportbezogener Leistung und der Förderung von Erziehungsfähigkeit (ebd., S. 67).

Eine wichtige neuzeitliche Quelle, die für manche als Geburtsstunde des modernen Business-Coachings steht, ist gemäß Garvey (ebd., S. 67) das 1974 erschienene Buch The Inner Game of Tennis von Timothy Gallwey. Es handelt vom Zustand der entspannten Konzentration («relaxed concentration») als Grundlage dafür, dass man im Tennisspiel sein volles Potenzial zur Entfaltung bringen kann. Eine weitere wichtige und auf Gallweys Buch aufbauende Quelle ist das erstmals 1988 von Sir John Whitmore veröffentlichte Buch Coaching for Performance. Im Zentrum dieses Werks steht das heute noch oft eingesetzte GROW-Modell, das dem Setzen von Zielen besondere Bedeutung beimisst (ebd., S. 68).

Zusammenfassend lassen sich aufgrund der ideengeschichtlichen, etymologischen und textlichen Wurzeln bereits an dieser Stelle einige wichtige Merkmale von Coaching festhalten. So soll es als Hilfeprozess Menschen helfen, ihr Potenzial zur Entfaltung zu bringen. Coaching soll Menschen also darin unterstützen, ihre Ziele zu erreichen und damit in ihnen wichtigen Dingen erfolgreich zu sein. Der Idee der Potenzialentfaltung einzelner Personen folgend, wird weiter nachvollziehbar, weshalb Coaching der personenorientierten und nicht etwa der Organisations-Beratung zugeordnet wird (Fietze 2011, S. 24; Schiersmann \& Thiel 2012, S. 8).

\subsubsection{Coaching als Personal- und Führungskräfteentwicklung}

In den 1970er Jahren ist Coaching in den USA als Instrument der modernen Personal- und Führungskräfteentwicklung aufgekommen. In Deutschland folgte eine ähnliche Entwicklung ab Mitte der 1980er Jahre (Böning 2005). Diese Entstehung des modernen Coachings sieht Geißler in sozioökonomischen Kontextveränderungen begründet. Er spricht von einem «globalisierungsbedingten Flexibilisierungs-, Innovations-, Kosten- und Qualitätsdruck privatwirtschaftlicher und öffentlicher Organisationen» (Geißler 2009, S. 93), der sich - als Grundlage auch für Coaching - erst einmal auf Führungskräfte übertragen habe.

Inhaltlich geht es in den USA zunächst um die entwicklungsorientierte Mitarbeiterführung und die Förderung von Nachwuchsführungskräften durch das 
Management (Böning 2005). Anders in Deutschland: Hier wird in erster Linie das Top-Management durch firmenexterne Coaches gecoacht. Inhaltlich geht es um Konflikte, Führungsprobleme, strategische Aufgaben und teilweise private Anliegen (z.B. Eheprobleme). In den frühen 1990er Jahren kommen dann auch in den USA organisationsexterne Coaches zum Einsatz. Führungskräfte der unteren und mittleren Ebene werden in der Folge durch organisationsinterne Coaches, oft Personalentwickler, begleitet - das Top-Management durch organisationsexterne Coaches.

Heute ist Coaching weitflächig fester Bestandteil systematisch betriebener Personal- und Führungskräfteentwicklung (ebd., S. 31 f.).

\subsection{Verbreitung und gesellschaftlicher Kontext}

Coaching-Angebot und Coaching-Nachfrage haben sich seit den 1990er Jahren im deutschsprachigen Raum und international ausgedehnt. Die Soziologin Beate Fietze, die die Entwicklung von Coaching über Jahre intensiv beobachtet hat, spricht von einer «breiten Marktetablierung» (Fietze 2010, S. 24). Oft wird auch von einem Coaching-Boom gesprochen (Böning \& Fritschle 2005; Gross \& Stephan 2012; Kotte et al. 2016, S. 6). Lindner (2011) schreibt gar von einem «Coaching-Wahn». Im Folgenden wird diese Verbreitung von Coaching noch etwas genauer beschrieben.

\subsubsection{Anzeichen eines Booms}

Als Belege für die starke Verbreitung von Coaching werden neben der Entwicklung von Angebot und Nachfrage (vgl. z. B. Bresser 2013; ICF 2012; Middendorf 2014; Seiger \& Künzli 2012; Stephan \& Gross 2013) auch Tagungsbesucher- oder Verbandsmitgliederzahlen angeführt (Greif 2008, S. 52). So zählte die International Coach Federation (ICF) - die weltweit größte berufliche Vereinigung von Coaches -2008 noch 10000 Mitglieder (ebd.) und 2016 bereits über $20000^{1}$.

Mit der Idee eines Coaching-Booms verbunden ist auch die Tatsache, dass sich Coaching nicht mehr auf den Profisport oder auf profitorientierte Großunternehmen beschränkt. Mittlerweile nehmen Menschen in unterschiedlichen Praxisfeldern und Funktionen Coaching in Anspruch (vgl. z.B. Wegener et al. 2016d; Wegener, Loebbert \& Fritze 2016a; Wegener, Loebbert \& Fritze 2016b). Zwei Bei-

1 www.coachfederation.org/about/landing.cfm?Item Number=741\&navItem Number=615 [12.8.2016]. 
spiele, die aufgrund ihrer politischen Bedeutung besonders interessant erscheinen, sind Coaching im Kontext von Arbeitsintegration (vgl. z. B. Vögeli 2015; Wegener 2016) und Coaching zur Förderung gesunder Lebensstile (vgl. z.B. Moore \& McBride 2014). Böning weist darauf hin, dass sich auch auf Coaching bezogene Inhalte, Settings und Methoden fortwährend weiterdifferenzieren. So werden in organisationalen Veränderungsprozessen sowohl Teams als auch Schlüsselpersonen mit Coaching begleitet (Böning 2005, S. 32).

\subsubsection{Gesellschaftlicher Kontext}

Soziologisch betrachtet, greifen die von Geißler thematisierten Entstehungsbedingungen weit über die Bedarfslage von Führungskräften hinaus. Die erhöhte Komplexität und Veränderungsgeschwindigkeit in westlichen Gesellschaften scheint nämlich, so die These, mit einem gesellschaftlich insgesamt steigenden Bedarf an Selbststeuerungskompetenz einherzugehen. Dieser Argumentation folgend, plädiert der Wirtschaftswissenschaftler Fredmund Malik dafür, Komplexität als Ressource zu nutzen, statt an ihr zu scheitern (Malik 2015). Coaching scheint in diesem gesellschaftlichen Zusammenhang eine mögliche Antwort zu sein.

Coaching wird auch von anderen Autoren und Autorinnen als Antwort auf gesellschaftliche Herausforderungen thematisiert (Greif 2014b; Wegener, Loebbert \& Fritze 2016c). So argumentiert Greif, in Anlehnung an Stelter (2014b) und Giddens (1991), dass Coaching als Reaktion auf die besonderen Herausforderungen der «hyperkomplexen spät- und postmodernen Gesellschaften» (Greif 2014b, S. 296) zu verstehen sei (ähnlich z.B. auch Böning \& Strikker 2014; Loebbert 2015, S. 133 ff.; Stelter 2014a; Wegener et al. 2016c). Fietze vertritt die Meinung, Veränderungen in der Arbeitswelt respektive damit verbundene Anforderungen an die individuelle Lebensführung habe Coaching zu einer derart nachgefragten Beratungsform werden lassen. Explizit verweist sie aber auch darauf, dass diese Veränderungen und deren Konsequenzen für den Einzelnen noch genauer zu erforschen seien (Fietze 2011, S. 26). Aus Sicht des Autors der vorliegenden Arbeit sollten insbesondere das konkrete Potenzial und die Begrenzung von Coaching erforscht werden. Die zentrale Leitfrage dabei wäre, für welche Situationen und Personen Coaching eine adäquate Maßnahme darstellt, auch wenn es nicht das Mittel der Wahl ist. 


\subsubsection{Coaching als marktorientierte oder professionelle Dienstleistung}

Die Tatsache, dass sich Coaching anhaltend verbreitet und ständig an Popularität gewinnt, wird nicht immer positiv gewertet. Es ist auch die Rede von «inflationärem Wortgebrauch» (Böning 2005; Fietze 2011, S. 24). Geißler (2009, S. 93) verweist auf die Gefahr, dass Coaching als Container- und Catch-all-Begriff auszubrennen drohe, da darin allem Platz gewährt werde. Tatsächlich besteht das Risiko, dass am Markt der Unmut wächst, weil unklar ist und bleibt, was professionelles Coaching ausmacht (Kühl 2008a, S. 268). So moniert Böning (2005, S. 33), dass Coaching seit seinem Erfolg in der Begleitung von Führungskräften mit allen möglichen Angeboten und Dienstleistungen in Verbindung gesetzt worden sei. Fachberatungen, mit etwas Feedback angereichert, werden als Coachings verkauft, um so - gemäß Böning - die Besonderheit von eigenen Angeboten herauszustreichen. Astrologische Beratung wird zu «Astro-Coaching», und Tanz-Schulungen werden $\mathrm{zu}$ «Dance-Coaching» (ebd.). Entsprechend intransparent und von unterschiedlicher Qualität sind indes auch die verschiedenen Coaching-Angebote. Dies führt dazu, dass Coaching an einer noch unklaren Identität leidet und dass von einem insgesamt tiefen Professionsniveau gesprochen wird (Fietze 2011, S. 24).

Coaching ist also zum einen ein hochgelobtes Beratungsformat, das den Nerv der Zeit trifft - weil es eine zeitgemäße Antwort auf aktuelle gesellschaftliche Herausforderungen liefert. Auf der anderen Seite wird der attraktiv klingende Begriff «Coaching» oft als Worthülse missbraucht, was zu einer schädlichen Verwässerung des Angebots führt.

$\mathrm{Ob}$ und wie sich Coaching weiter etablieren wird, ist aus Sicht des Verfassers der vorliegenden Arbeit derzeit ungewiss. Möglich erscheinen zumindest zwei Szenarien. Erstens: Coaching bleibt eine rein markgesteuerte Dienstleistung, die entsprechenden Dynamiken und Interessen unterworfen ist. Denkbar ist zweitens, dass Coaching sich zu einer professionellen Dienstleistung oder zu einer Profession weiterentwickelt. Letzteres ist allerdings nur möglich, wenn flächendeckend hohe Qualitätsstandards definiert und konsequent eingefordert werden.

\subsection{Professionalisierung}

Im Folgenden wird der auf Coaching bezogene Professionalisierungsdiskurs abgebildet. Dazu werden Professionalisierungsbedürftigkeit und der Stand der gegenwärtigen Professionalisierung von Coaching diskutiert. Dabei soll insbesondere 
sichtbar werden, welche Rolle die Wissenschaft für die weitere Professionalisierung von Coaching spielt.

\subsubsection{Professionalisierungsbedürftigkeit}

Ob Coaching zu einer professionellen Dienstleistung oder einer Profession wird, hängt, professionssoziologisch betrachtet, davon ab, welcher Problemtypus mit Coaching bearbeitet wird (Fietze 2011): Je existenzieller der Problemtypus und je schwerwiegender die Folgen einer nicht professionellen Bearbeitung des Problemtypus, desto wichtiger ist es, entsprechende berufliche Tätigkeiten zu professionalisieren.

Der mit Coaching adressierte Problemtypus kann im Sinne des Anspruchs erfolgreicher Selbststeuerung in einer zunehmend komplexen und schnell sich verändernden Gesellschaft aus Sicht des Verfassers dieser Arbeit in der Tat als existenziell bewertet werden. Wem es heute nicht gelingt, mit Veränderung erfolgreich umzugehen, der läuft Gefahr, gesellschaftlich marginalisiert zu werden. Persönliche Lern- und Entwicklungsprozesse werden also zur Grundlage erfolgreicher Lebensführung - dies im privaten wie auch im beruflichen Bereich. Die Nachfrage nach Coaching wird voraussichtlich weiter steigen, da es genau diese Herausforderung adressiert. Zudem werden weder die Geschwindigkeit, mit der sich die Welt verändert, noch die Komplexität, die damit einhergeht, abnehmen eher dürfte das Gegenteil eintreffen.

Die Professionalisierungsbedürftigkeit von Coaching sieht der Soziologe Kühl, in Ergänzung zur Argumentation von Fietze, in den Folgen nicht professionell durchgeführter Coachings begründet. Sein zentrales Argument lautet, dass es Coaches in ihren «quasitherapeutischen» Coaching-Situationen immer wieder misslingt, therapeutische Überweisungsfälle zu erkennen. Die Gesundheit von Klienten und Klientinnen mit psychischen Problemen kann also im schlimmsten Fall durch nicht professionell durchgeführte Coachings verschlechtert werden. Eine weitere Gefahr sieht Kühl darin, dass nicht professionell arbeitende Coaches psychisch labile Zustände ihrer Kunden und Kundinnen missbrauchen und Abhängigkeiten schaffen (Kühl 2008a, S. 269). Im Sinne dieser Argumentation gibt es bereits eine Fülle empirischer Studien, die sich mit negativen Effekten von Coaching beschäftigt (vgl. z. B. Bachmann 2015; Oellerich 2016; Schermuly 2014; Schermuly et al. 2014) und den empirischen Gehalt dieser These untermauern. 


\subsubsection{Stand der Professionalisierung}

Folgt man Böning und Fritschle (2005, S. 22-25), durchläuft Coaching bereits seit geraumer Zeit einen Professionalisierungprozess. Dies machen die beiden Autoren an folgenden Aspekten fest:

- Erstens findet bereits eine zielgruppenspezifische und methodisch differenzierte Anwendung von Coaching statt;

- zweitens steigen die Qualitätsanforderungen in der Praxis;

- drittens wird eine beginnende Markttransparenz beobachtet;

- viertens erfolgt eine zunehmende Standardisierung in der Praxis und Ausbildung;

- fünftens intensiviert sich die Forschung zu Coaching;

- sechstens gibt es immer mehr Kongresse und Fachtagungen;

- siebtens ist eine zweite Coach-Generation nachgerückt.

Ähnlich geht Fietze (2011, S. 25) davon aus, dass eine Professionalisierung von Coaching bereits im Gange sei. Sie begründet ihre Position mit dem Verweis auf drei zentale Coaching-Phasen und damit verbundene Professionalisierungsaktivitäten. Die «stürmische Marktetablierung» als erste Phase begann Anfang der 1990er Jahre. Ende des 20. Jahrhunderts setzte die Phase der «Entstehung der kollegialen Selbstorganisation» ein. Diese Phase ist gekennzeichnet durch die Gründung einer Vielzahl von Berufsverbänden. ${ }^{2}$ Die dritte Coaching-Phase sieht Fietze in der «Intensivierung des Wissenschaftsbezugs». Sie ist erkennbar an einer rasant steigenden Zahl wissenschaftlicher Veröffentlichungen (Fietze 2011, S. 25). Besonders intensiviert hat sich diese dritte Phase, die im professionssoziologischen Diskurs als zentraler Hinweis auf eine sich akzentuierende Professionalisierung verstanden wird (ebd., S. 25), seit 2010. Und trotz dieses überproportional starken Anstiegs muss der Stand wissenschaftlicher Publikationen als ingesamt noch bescheiden bewertet werden (vgl. z.B. Bachkirova, Sibley \& Myers 2015; Greif 2014b; Möller \& Kotte 2011) - Letzteres sowohl in Bezug auf belastbare, randomisiert kontrollierte Interventionsstudien (RCT) zur Wirksamkeit von Coaching (Greif 2016) als auch bezogen auf Untersuchungen zu Coaching-Prozessen, die

2 Interessant in diesem Zusammenhang ist auch der Roundtable der Coachingverbände, eine Plattform von dreizehn deutschen Coaching-Verbänden. Sie veröffentlichten unter diesem gemeinsamen Dach im März 2015 das Positionspapier «Qualität und Professionalität», in dem einheitliche Coaching-Standards festgehalten werden (www. coachfederation.de/professionalisierung/roundtable-der-coachingverbaende.html [20.4.2016]). 
Auskunft darüber geben, was Coaching ist und ausmacht (Geißler 2016; Myers 2014).

Trotz der Kritik einer noch bescheidenen Forschungslage gibt es somit, wenn wir den Argumentationen von Böning, Fritschtle und Fietze folgen wollen, Hinweise darauf, dass die Professionalisierung von Coaching läuft.

\subsubsection{Professionalisierungsherausforderungen}

Wenn Coaching als personenorientiertes Beratungsformat nicht weiter verwässern, sondern zu einer anerkannten professionellen Dienstleistung oder gar Profession werden soll, müssen aus Sicht des Autors dieser Arbeit die folgenden Professionalisierungsherausforderungen ernst genommen werden:

- Coaching muss als Beratungsformat klar bestimmt und von anderen Beratungsangeboten abgegrenzt werden.

- Qualitätsstandards müssen formuliert und vor allem eingefordert werden.

- Drittens braucht es eine hochwertige Coaching-Forschung, die Auskunft sowohl über die Wirksamkeit als auch über die Wirkweise von Coaching gibt (vgl. auch Wegener 2013).

Ob die Professionalisierung von Coaching weiter voranschreitet, hängt in einem hohen Maß davon ab, ob und wieweit es den zentralen institutionellen Akteuren gelingen wird, Coaching als professionelles Beratungsformat $\mathrm{zu}$ positionieren (Wegener 2013). Zu diesen Akteuren zählen Anbieter und Nachfrager, Berufsverbände wie auch Forschungs- und Weiterbildungsinstitutionen und nicht zuletzt die Medien, die das öffentliche Bild von Coaching mitgestalten.

\subsection{Begriffsbestimmung}

Eine systematische Auswertung gängiger Definitionen zeigt, dass es keinen Konsens gibt, was Coaching sei (Bachkirova \& Kauffman 2009). Dies überrascht aufgrund der unklaren Identität von Coaching und der unüberschaubaren Zahl an Angeboten am Markt nicht weiter. Die Klärung der Frage, was Coaching sei respektive sein solle, wird aber aufgrund ihrer Relevanz andauern. Sowohl der theoretische Diskurs als auch empirische Studien, mittels deren untersucht wird, was Coaching ausmacht, sind hier von Bedeutung. 
Im Sinne eines pragmatischen Vorgehens scheint es angebracht, einige aktuell diskutierte Definitionen vorzustellen und auf dieser Grundlage eine Arbeitsdefinition für die vorliegende Arbeit herzuleiten. Die im Folgenden abgebildete Auswahl an zu diskutierenden Coaching-Definitionen erfolgte im ersten Schritt intuitiv und im weiteren Rechercheverlauf reflektiert mit Bezug auf folgende Merkmale:

- Erstens handelt es sich um Definitionen, denen der Autor der vorliegenden Arbeit in wissenschaftlichen Arbeiten mehrfach begegnet ist.

- Zweitens stammen die Definitionen von Autoren und Autorinnen, die im wissenschaftlichen Diskurs eine hohe Anerkennung genießen und/oder in Bezug auf die Entstehungsgeschichte von Coaching seitens der Wissenschaft als wichtig erachtet werden.

- Drittens wurden die Definitionen von Autoren und Autorinnen verfasst, die Monografien zu Coaching verfasst haben.

- Viertens haben diese Definitionen den Autor der vorliegenden Arbeit deshalb in besonderer Weise angesprochen, weil sie in Bezug auf das Kriterium der Unterscheidung von Coaching gegenüber anderen personenorientierten Beratungsformen - wie etwa der Psychotherapie oder der Mediation - fruchtbar erscheinen.

Die folgende Darstellung der Definitionen erfolgt eingebettet in kurze Darstellungen ihrer Autoren und Autorinnen. Die Auswertung der Definitionen und ihre Überführung in die Coaching-Definition, die der vorliegenden Arbeit zugrunde gelegt wird, geschieht im anschließenden Kapitel.

\subsubsection{Ausgewählte Definitionen von Coaching}

Die erste Coaching-Definition, die ich hier anführen möchte, stammt von der deutschsprachigen Coaching-Pionierin Astrid Schreyögg. Gemäß Greif (2015b) stellt sie als reflektierte Praktikerin in ihren Veröffentlichungen die stärksten Wissenschaftsbezüge her. Ihr Klassiker Coaching: eine Einführung für Praxis und Ausbildung liegt bereits in siebter Auflage vor (Schreyögg 2012). Auf andere Autoren und Autorinnen Bezug nehmend, versteht Schreyögg unter Coaching Folgendes:

«Anhand der Publikationen bekannter Autoren im deutschsprachigen Raum, die sich zum Thema Coaching geäußert haben (Böning \& Fritschle 2005; Kühl 
2008b; Looss 2006; Rauen 2005 u.a.), zeichnet sich ab, dass es eine innovative Form der Personalentwicklung für Menschen mit Managementfunktionen darstellt [...]. Außerdem handelt es sich um eine Dialogform, bei der Freud und Leid im Beruf verhandelt werden. In diesem Sinn kann Coaching auch als Therapie gegen berufliches Leid, aber auch als Maßnahme zur Förderung eines erfolgreichen und glücklichen beruflichen Daseins bezeichnet werden, wie es heute gelegentlich unter dem Begriff 〈Lifecoaching〉 beschrieben wird (Buer \& Schmidt-Lellek 2008). Diese Begriffsverwendungen entsprechen übrigens auch dem Verständnis internationaler Publikationen, wie sie sich neuerdings etwa in Magazinen der Academy of Management manifestieren (Segers et al. 2011). Dabei zielt Coaching idealerweise auf eine maximale Selbstgestaltung im Berufsleben. Und wo die Möglichkeit zur Selbstgestaltung vorübergehend oder längerfristig verloren ging, soll sie durch Coaching wiedergefunden werden.» (Schreyögg 2012, S. 66)

Die zweite Definition stammt vom Arbeits- und Organisationspsychologen Siegfried Greif. Er zählt zu den meistzitierten Coaching-Forschern im deutschsprachigen Raum. Als einer von wenigen deutschsprachigen Autoren hat er auch Beiträge zu Coaching in wichtigen englischen Publikationen veröffentlicht, weshalb er auch im englischsprachigen Raum rezipiert wird (vgl. dazu z. B. Bachkirova et al. 2015; Greif 2013; Greif 2017). Zu Greifs wichtigsten Forschungsschwerpunkten zählt das aus der Psychotherapie-Forschung adaptierte Wirkfaktorenmodell (Greif 2008; Greif 2015; Greif, Schmidt \& Thamm 2010; Greif, Schmidt \& Thamm 2012) und ein darauf Bezug nehmendes Coaching-Evaluationsmodell (Greif 2008; Greif 2013; Greif 2014a). Die häufig zitierte Coaching-Definition von Greif lautet so:

«[...] eine intensive und systematische Förderung ergebnisorientierter Problemund Selbstreflexionen sowie Beratung von Personen oder Gruppen zur Verbesserung der Erreichung selbstkongruenter Ziele oder zur bewussten Selbstveränderung und Selbstentwicklung. Ausgenommen ist die Beratung und Psychotherapie psychischer Störungen.» (Greif 2008, S. 69)

Die dritte Definition stammt vom international renommierten Coaching-Wissenschaftler Anthony Grant. Er wurde bekannt durch seine Forderung nach «Evidence-based Coaching» (Stober \& Grant 2006) und damit verbunden durch die wissenschaftliche Verortung von Coaching in der durch ihn geprägten «Coaching-Psychologie». Gemeinsam mit Stober definiert Grant Coaching wie folgt: 
«A collaborative and egalitarian relationship between a coach, who is not necessarily a domain-specific specialist, and a client, which involves a systematic process that focuses on collaborative goal setting to construct solutions and employ goal attainment process with the aim of fostering the on-going self-directed learning and personal growth of the client.» (Grant \& Stober 2006, S. 2)

Elaine Cox und Tatiana Bachkirova, zwei renommierte britische Coaching-Forscherinnen der Oxford Brookes University, haben das weltweit erste Doktoranden-Programm für Coaching etabliert. Gemeinsam mit David Clutterbuck definieren sie Coaching folgendermaßen:

«Coaching could be seen as a human development process that involves structured, focused interaction and the use of appropriate strategies, tools, and techniques to promote desirable and sustainable change for the benefit of the coachee and potentially other stakeholder.» (Cox et al. 2010, S. 1)

Sir John Whitmore, dessen Buch Coaching for Performance (1992) - neben The Inner Game of Tennis von Timothy Gallwey (1974) - zu den Klassikern der Coaching-Praxisliteratur zählt, versteht unter Coaching dies:

"Coaching is unlocking people's potential to maximize their own performance. It is helping them to learn rather than teaching them.» (Whitmore 1992, S. 10)

Die zweitletzte Definition, die hier aufgeführt wird, stammt vom Erziehungswissenschaftler und Begründer der Organisationspädagogik Harald Geißler. Geißler ist ein international anerkannter Pionier in der Entwicklung und Beforschung digital gestützter Coaching-Formate. Darüber hinaus hat er ein sinnerschließendes Interpretationssystem entwickelt, mittels dessen der Sinn einzelner Kommunikationsentscheide von Coach und Kunde auf präzise Weise rekonstruiert werden kann (Geißler 2016). Geißler definiert Coaching wie folgt:

«Coaching definiert sich zum einen durch die Offenheit möglicher Lösungen einer vorliegenden Kundenproblematik [...] und zum anderen durch die Aufgabe des Coaches, bei der Erfassung und Analyse der vorliegenden Problematik und Entwicklung einer passenden Lösung konsequent die Selbstbestimmung (Selbstwirksamkeit) des Kunden anzusprechen [...]. Diese Aufgabe muss ausgerichtet sein auf den Problemlösungsunterstützungsbedarf des Kunden [...]. Er setzt sich aus drei Teilbedarfen zusammen, nämlich aus dem Aufklärungs-, Umsetzungs- und Transformationsunterstützungsbedarf. Mit Bezug auf diese Bedarfsstruktur begründet sich die Zielstruktur des Coachings. Weiterhin ist für Coaching charakteristisch, 
dass der Problemunterstützungsbedarf des Kunden immer vom Erkenntnisstandpunkt des jeweils erreichten Stands der Problemlösung bestimmt werden muss und dass deshalb zwischen dem Problemlösungsunterstützungsbedarf und dem Entwicklungsstand der Problemlösung ein zirkulärer Zuammenhang besteht [...]. Und schließlich ist Coaching ein Prozess, der mit zeitlich eng begrenzten Ressourcen zurechtkommen muss [...].»(Geißler 2015, S. 25)

Abschließend wird noch die Definition von Michael Loebbert aufgeführt, einem deutschen Coaching-Theoretiker, Coaching-Weiterbildungsverantwortlichen und langjährigen Executive Coach. Nach ihm ist Coaching eine «persönliche Form der Prozessberatung» (Loebbert 2015, S. 3, 20 ff.). Loebbert lehnt sich dabei an Schein (2010) an und bezieht sich in besonderer Weise auf das, «was sich zwischen einem Helfer und dem Menschen, dem geholfen wird, abspielt». Weiter präzisiert er, dass sich Coaching im Kontext seiner Betrachtung immer «auf den Leistungsund Handlungsprozess von Personen» bezieht (Loebbert 2015).

\subsubsection{Auswertung der Definitionen}

In Tabelle 1.1 werden die inhaltlichen Bestimmungsmerkmale der vorgestellten Definitionen abgebildet. Mit Blick auf ihre weitere Verwendung werden sie in Klammern nummeriert. Die vom Autor später präsentierte Definition bezieht sich auf eine Auswahl dieser Bestimmungsmerkmale (in Tabelle 1.1 grau hinterlegt). Zudem enthält die Definition ein darüber hinausgehendes Bestimmungsmerkmal, das gleich noch vorgestellt wird. Es ist der Anspruch an die so hergeleitete Definition, Coaching einerseits klar zu bestimmen und zugleich von anderen Beratungsformaten wie Psychotherapie, Fachberatung oder psychosozialer Beratung abzugrenzen. 
Tabelle 1.1 Bestimmungsmerkmale der ausgewählten Coaching-Definitionen

\begin{tabular}{|c|c|}
\hline $\begin{array}{l}\text { Autoren/ } \\
\text { Autorinnen }\end{array}$ & Bestimmungsmerkmale von Coaching \\
\hline Schreyögg & 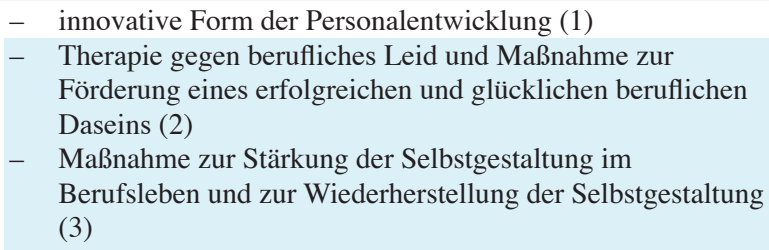 \\
\hline Greif & 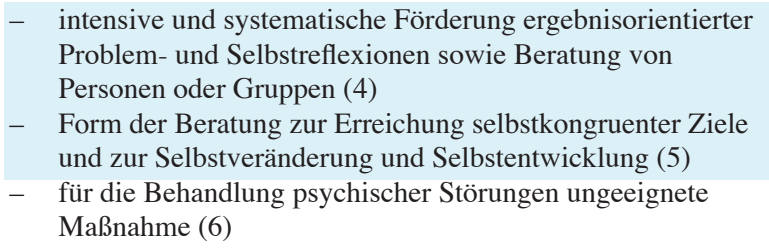 \\
\hline Grant & 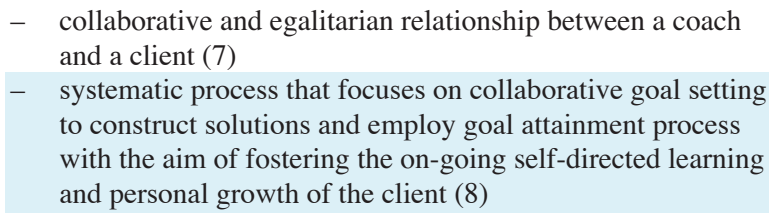 \\
\hline $\begin{array}{l}\text { Bachkirova, Cox } \\
\text { und Clutterbuck }\end{array}$ & $\begin{array}{ll}\text { - } & \text { human development process (9) } \\
- & \text { structured, focused interaction and use of appropriate } \\
& \text { strategies, tools, and techniques (10) } \\
- & \text { promoting desirable and sustainable change for the benefit } \\
& \text { of the coachee and potentially other stakeholder (11) }\end{array}$ \\
\hline Whitmore & $\begin{array}{l}\text { - unlocking people's potential to maximize their performance } \\
\text { (12) } \\
\text { - } \text { helping to learn rather than teaching (13) }\end{array}$ \\
\hline Geißler & $\begin{array}{ll}- & \text { bezogen auf die Lösungen offener Problemlösungsprozess } \\
& (14) \\
- & \text { konsequent auf die Selbstbestimmung (Selbstwirksamkeit) } \\
& \text { bezogener Prozess (15) } \\
- & \text { auf den aktuellen Problemlösungsunterstützungsbedarf } \\
& \text { bezogener Prozess (Aufklärungs-, Umsetzungs- und } \\
& \text { Transformationsunterstützungsbedarf) (16) } \\
- & \text { zeitlich eng begrenzter Prozess (17) }\end{array}$ \\
\hline Loebbert & $\begin{array}{ll}\text { - } & \text { persönliche Prozessberatung (18) } \\
\text { - } & \text { auf den Leistungs- und Handlungsprozess der Kundinnen } \\
& \text { und Kunden bezogene Beratung (19) }\end{array}$ \\
\hline
\end{tabular}


Neben den grau hinterlegten Bestimmungsmerkmalen wird in der gleich anschließend präsentierten Definition von Coaching auch das berücksichtigt, was Schreyögg an anderer Stelle als zentrale Wirkung eines Coachings bezeichnet. Dabei spricht sie davon, dass Kunden und Kundinnen in einem Coaching, bezogen auf ihre Anliegen, «funktionale Deutungs- und Handlungsmuster» (Schreyögg 2012, S. 194, 200 ff.) ausbilden müssten, um in ihrem Handeln (wieder) erfolgreich zu sein. Entsprechend lautet nun unsere Coaching-Definition für die vorliegende Arbeit:

Coaching als persönliche Form der Prozessberatung (18) unterstützt Klientinnen und Klienten dabei, unter Berücksichtigung ihrer Talente und Fähigkeiten (12) selbstkongruente Leistungs- und Handlungsziele (2, 3, 5, 9) zu identifizieren und/ oder zu erreichen. Zu diesem Zweck werden Klientinnen und Klienten von Coaches dazu angeregt, notwendige Lernprozesse $(4,8,9,13)$ zu vollziehen, um zur Verbesserung ihrer Selbststeuerung (15) und bezogen auf ihre Anliegen funktionale Deutungs- und Handlungsmuster auszubilden. Die Überwindung psychischer Störungen (6) sowie reine Wissensvermittlung sind nicht Gegenstand von Coaching.

\subsection{Forschungsvorhaben}

Im Folgenden geht es darum, Einblick in das vorliegende Forschungsvorhaben zu geben.

\subsubsection{Forschungsdesiderat}

Wie erwähnt, kommt der Wissenschaft in der Professionalisierung von Coaching eine besondere Bedeutung zu. Fietze unterscheidet dazu zwischen einer Orientierungs- und einer Legitimationsfunktion der Forschung (2011, S. 25 f.). Die Orientierungsfunktion von Forschung verweist darauf, dass Coaching durch Forschung inhaltlich näher bestimmt und damit als Forschungsgegenstand etabliert wird. Die Legitimationsfunktion verweist darauf, dass praktizierende Coaches und Coaching als Profession durch Forschung zu mehr sozialer Autorität gelangen und in ihrem Autonomieanspruch gestärkt werden. Bezogen auf die im Coaching-Diskurs als dominant bewertete Nachfrageseite (d.h. vor allem die Unternehmen, die Coaching nachfragen), erscheint ein solcher Ausgleich als sehr wichtig.

Was die inhaltliche Ausrichtung der Forschung betrifft, spricht Fietze sinnbildlich vor allem davon, «Licht in die Blackbox des Beratungsprozesses [zu] bringen 
und vor allem diejenigen Aspekte [zu] beleuchten, die durch professionelles Handeln beeinflusst werden und durch empirische Erkenntnisse optimiert werden können [...]» (ebd., S. 28). Diese auf die Wirkweise von Coaching bezogene Forschung ist für Praktikerinnen und Praktiker besonders wertvoll, da sie eine empirisch fundierte Handlungsorientierung gewährleistet. Fietze spricht auch von einer «Entscheidungshilfe in Zweifelsfragen», von «Risikominderung ihrer professionellen Interventionen» und von «Entlastung ihrer Handlungsverantwortung» (ebd., S. 29). In dieser Absicht möchte auch die vorliegende Arbeit einen Beitrag leisten - also sowohl für die Wissenschaft als auch für die Praxis relevante Ergebnisse produzieren.

Zur Verortung der Arbeit ist es hilfreich, auf eine zentrale Unterscheidung zwischen zwei Varianten der wirkungsbezogenen Coaching-Forschung einzugehen. Die erste und zugleich weiter fortgeschrittene Variante hat zum Ziel, die Wirksamkeit von Coaching nachzuweisen (vgl. dazu etwa Möller \& Kotte 2011) - dies etwa im Vergleich zu anderen Formaten wie Training oder Psychotherapie. Im weiteren Verlauf wird bei dieser Art der Forschung von ergebnisorientierter Coaching-Forschung oder Coaching-Ergebnisforschung gesprochen. Die zweite, weniger etablierte Variante der wirkungsbezogenen Coaching-Forschung (ebd.) hat zum Ziel, mehr darüber in Erfahrung zu bringen, wie Coaching-Prozesse verlaufen. Dabei interessieren etwa Faktoren, die für den Erfolg von Coaching relevant sind - so Verhaltensweisen von Coaches, die den Coaching-Verlauf unmittelbar positiv beeinflussen (z.B. wertschätzendes oder emotional unterstützendes Verhalten; vgl. Greif 2016, S. 171). Im weiteren Verlauf dieser Arbeit wird bezüglich dieser zweiten Art der Forschung von prozessorientierter Coaching-Forschung oder Coaching-Prozessforschung gesprochen.

Das Forschungsneuland, das mit der vorliegenden Arbeit betreten wird, verortet sich im Kontext der prozessorientierten Coaching-Forschung. Als forschungsleitender Startpunkt interessierte zunächst die Frage, ob es so etwas wie «bedeutsame Momente» im Coaching gibt und wie solche Momente durch die Untersuchung konkreter Coaching-Prozesse erforscht und beschrieben werden könnten. Die sorgfältige Rekonstruktion und Differenzierung dieses zunächst eher intuitiv begründeten Forschungsdesiderats führt im ersten Teil der Arbeit zu einer fruchtbaren Auswertung des aktuellen Coaching-Forschungsstands. Erweitert durch einen Exkurs in die Psychotherapie-Forschung, ist es so nämlich gelungen, wichtige Erkenntnisse für die weitere Ausrichtung der Coaching-Prozessforschung zu formulieren und damit verbunden eine prozessorientierte Coaching-Forschungslandkarte zu skizzieren. Im Anschluss daran wird im zweiten Teil der Arbeit im Sinne eines explorativen Vorgehens ein zweistufiges Verfahren zur Identifikation und Analyse «bedeutsamer Momente» entwickelt, exemplarisch auf einen konkreten Coaching-Fall angewandt und ausgewertet. 


\subsubsection{Forschungsfragen}

Wie erwähnt, besteht das Ziel dieser Arbeit darin, ein methodisches Verfahren zu entwickeln, mittels dessen «bedeutsame Momente» im Coaching durch die Analyse faktischer Coaching-Verläufe identifiziert und mit Blick auf ihre besondere Beschaffenheit rekonstruiert werden können. Aus diesem Forschungsziel ergaben sich drei Forschungsfragen:

- Was sind im Sinne eines theoretischen Vorverständnisses «bedeutsame Momente» im Coaching? (Forschungsfrage 1)

- Wie lassen sich «bedeutsame Momente» im Coaching im Verlauf von faktischen Coaching-Sitzungen identifizieren? (Forschungsfrage 2)

- Wie können «bedeutsame Momente» im Coaching im Verlauf von faktischen Coaching-Sitzungen vertiefend analysiert werden? (Forschungsfrage 3)

\subsubsection{Untersuchungsbestimmende Vorentscheidungen}

Im Folgenden werden Entscheidungen beschrieben, die der Verfasser der vorliegenden Arbeit bereits früh im Forschungsprozess gefällt hat.

\section{Analyse faktischer Coaching-Verläufe}

Ein erster wichtiger Entscheid bestand darin, ein Forschungsdesign zu entwickeln, mittels dessen «bedeutsame Momente» im Coaching auf der Grundlage faktischer Coaching-Verläufe erforscht werden sollten. Dieser methodische Entscheid fiel unter anderem in Auseinandersetzung mit einer Reihe von Studien der Forschergruppe um Erik de Haan (Day et al. 2008; de Haan 2008c; de Haan 2008b; de Haan et al. 2010b; de Haan et al. 2010a; de Haan, Culpin \& Curd 2011; de Haan \& Nieß 2012; de Haan \& Nieß 2014). In diesen Untersuchungen, deren Grundlagen und Ergebnisse in Kapitel 2 erörtert werden, wurden in Interviews und schriftlichen Umfragen Coaches und Kunden befragt, was ihnen in ihrer Erinnerung und bezogen auf real erlebte Coachings als «critical moments» geblieben sei. Trotz einiger Vorzüge, die an späterer Stelle diskutiert werden, besteht eine Kritik an diesem methodischen Vorgehen darin, dass dabei «nur» subjektive Erlebensweisen, nicht aber Informationen zum von außen beobachtbaren, faktischen Verlauf entsprechender Momente gewonnen und ausgewertet werden. Eine zweite Kritik bezieht sich darauf, dass in Befragungen erhobene Daten wahrnehmungs- und gedächtnisbedingt nicht selten verzerrt sind. Verzerrt sind sie oft auch aufgrund des Phänomens der «sozialen Erwünschtheit»: Interviewte oder schriftlich befragte 
Personen beantworten Fragen zuweilen so, wie es aus ihrer Sicht von ihnen erwartet wird, statt ihr tatsächliches Erleben und Empfinden zum Ausdruck zu bringen.

Ein wichtiger Vorteil, der sich aus dem methodischen Entscheid ergibt, anstelle von Interviews oder schriftlichen Befragungen faktische Coaching-Prozesse auszuwerten, ist der hohe mögliche Grad an Präzision in der Beantwortung der Frage, was «bedeutsame Momente» im Coaching sind und wie sie entstehen. Damit ist die Tatsache gemeint, dass auf der Grundlage aufgezeichneter Coaching-Sitzungen bis auf die Ebene einzelner Sprechakte Erkenntnisse gewonnen werden können. Im Zusammenhang mit der Analyse «bedeutsamer Momente» im Coaching wird dieser Vorteil - wie gleich anschließend und in Kapitel 4 noch genauer beschrieben wird - ausgeschöpft.

\section{Analyse einzelner Kommunikationsentscheidungen}

Eine zweite Vorentscheidung dieser Arbeit bestand darin, «bedeutsame Momente» im Coaching mithilfe des von Geißler entwickelten Systems zur sinnerschließenden Interpretation (Geißler 2016) und Analyse faktischer Coaching-Verläufe zu untersuchen.

Mit diesem Entscheid rückten die einzelnen Sprechakte von Coach und Kunden und deren verborgener Sinn in den Vordergrund der Untersuchung. Im Forschungsansatz von Geißler - der in den Folgekapiteln noch ausführlich vorgestellt wird geht es nämlich darum, Coaching als «Kommunikationsgattung» zu bestimmen. Dazu beabsichtigte Geißler, alle möglichen Kommunikationsentscheidungen, die sich den Coaches und Kunden in einem Coaching anbieten, zu rekonstruieren und damit verbundene Nutzungshäufigkeiten zu errechnen. Als Grundlage für die Umsetzung dieses Vorhabens entwickelte Geißler ein Kategoriensystem, mittels dessen einzelne Sprechakte im Sinne von «Kommunikationsentscheidungen» durch elf unterschiedliche Entscheidungsdimensionen präzise bestimmt werden können.

Die Vermutung, dass sich der Ansatz von Geißler für die Untersuchung «bedeutsamer Momente» im Coaching eignen würde, musste im Verlauf der Untersuchung erst noch überprüft werden. Zu Beginn der Arbeit wurde diese Vermutung jedoch durch die Überlegung gestützt, dass sich «bedeutsame Momente» im Coaching über besondere respektive sich besonders verändernde Kommunikationsentscheidungen der Kundinnen und Kunden sowie der Coaches rekonstruieren lassen. 


\subsubsection{Aufbau}

Die vorliegende Arbeit besteht neben dem vorliegenden Einleitungs- aus vier weiteren Kapiteln.

In Kapitel 2 wird der Stand der Coaching-Forschung ausgewertet. Verbunden mit einem Exkurs in die Psychotherapie-Forschung, resultiert daraus als erstes zentrales Ergebnis dieser Arbeit und als Vorschlag für die Erweiterung der gegenwärtigen Coaching-Forschung eine prozessorientierte Forschungslandkarte für Coaching. Als zweites Ergebnis, mit der Entwicklung dieser Forschungslandkarte verbunden, ist die weitere Eingrenzung des Forschungsdesiderats, das mit dieser Arbeit bearbeitet werden soll, sowie eine erste Bestimmung von relevanten theoretischen und methodologisch-methodischen Aspekten dazu zu nennen.

In Kapitel 3 wird das Forschungsdesign zur Untersuchung «bedeutsamer Momente» im Coaching weiter präzisiert. Es wird zunächst die Frage beantwortet, was im Sinne eines sensibilisierenden Konzepts unter «bedeutsamen Momenten» im Coaching verstanden wird (Forschungsfrage 1). Anschließend wird im Sinne eines zu überprüfenden Vorschlags beschrieben, wie «bedeutsame Momente» im Coaching aufgrund der Analyse faktischer Coaching-Verläufe zuverlässig identifiziert (Forschungsfrage 2) und im Sinne der Bestimmung ihrer besonderen Charakteristika präzise analysiert (Forschungsfrage 3) werden können.

In Kapitel 4 werden die in Kapitel 3 beschriebenen Analyseschritte exemplarisch erprobt. Grundlage für diese Anwendung des methodischen Designs bildet ein drei Sitzungen umfassendes, telefonisch durchgeführtes Coaching.

Im letzten Kapitel schließt sich der Bogen; es wird diskutiert, inwiefern und mit welcher Qualität die drei gestellten Forschungsfragen beantwortet wurden. Im darauf folgenden Ausblick wird geklärt, wie das vorgestellte Forschungsdesign weiterentwickelt werden kann und welche weiterführenden Forschungsfragen sich stellen.

\subsubsection{Geschlechtergerechte Sprache}

Im Sinne einer geschlechtergerechten Sprache werden in der vorliegenden Arbeit zuweilen weibliche und männliche Formen abwechselnd oder in Kombination verwendet, z. B. Forscherinnen, Lehrer und Wissenschaftlerinnen -, oder aber es werden beide Formen genannt: Forscher und Forscherinnen usw. Exklusiv männliche oder weibliche Schreibweisen werden nur gewählt, wenn auf reale Personen des entsprechenden Geschlechts direkt Bezug genommen wird. 
Open Access Dieses Kapitel wird unter der Creative Commons Namensnennung 4.0 International Lizenz (http://creativecommons.org/licenses/by/4.0/deed.de) veröffentlicht, welche die Nutzung, Vervielfältigung, Bearbeitung, Verbreitung und Wiedergabe in jeglichem Medium und Format erlaubt, sofern Sie den/die ursprünglichen Autor(en) und die Quelle ordnungsgemäß nennen, einen Link zur Creative Commons Lizenz beifügen und angeben, ob Änderungen vorgenommen wurden.

Die in diesem Kapitel enthaltenen Bilder und sonstiges Drittmaterial unterliegen ebenfalls der genannten Creative Commons Lizenz, sofern sich aus der Abbildungslegende nichts anderes ergibt. Sofern das betreffende Material nicht unter der genannten Creative Commons Lizenz steht und die betreffende Handlung nicht nach gesetzlichen Vorschriften erlaubt ist, ist für die oben aufgeführten Weiterverwendungen des Materials die Einwilligung des jeweiligen Rechteinhabers einzuholen.

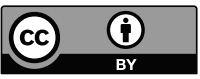

